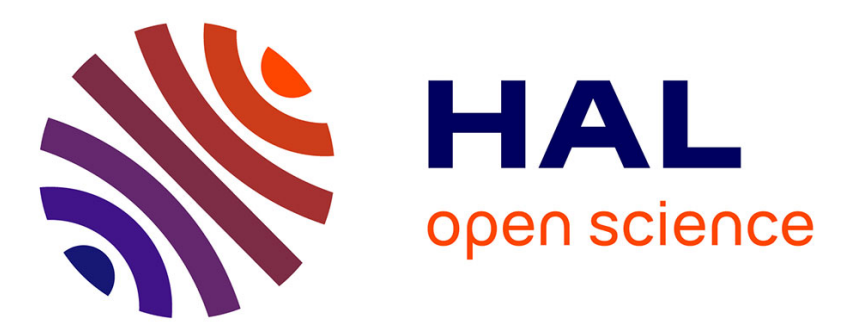

\title{
Explicit solutions to dynamic portfolio choice problems: A continuous-time detour
}

François Legendre, Djibril Togola

\section{To cite this version:}

François Legendre, Djibril Togola. Explicit solutions to dynamic portfolio choice problems: A continuous-time detour. 2015. hal-01117787v2

\section{HAL Id: hal-01117787}

\section{https://hal-enpc.archives-ouvertes.fr/hal-01117787v2}

Preprint submitted on 6 Jul 2015

HAL is a multi-disciplinary open access archive for the deposit and dissemination of scientific research documents, whether they are published or not. The documents may come from teaching and research institutions in France or abroad, or from public or private research centers.
L'archive ouverte pluridisciplinaire HAL, est destinée au dépôt et à la diffusion de documents scientifiques de niveau recherche, publiés ou non, émanant des établissements d'enseignement et de recherche français ou étrangers, des laboratoires publics ou privés. 


\title{
Explicit solutions to dynamic portfolio choice problems: A continuous-time detour
}

\author{
François Legendre* $\quad$ Djibril Togola ${ }^{\dagger}$
}

May 26, 2015

\begin{abstract}
Recently, many academic researchers have implemented different numerical procedures to solve a dynamic portfolio choice problem especially in incomplete markets. The subsequent numerical results are sometimes significantly different from one paper to another. Thus, they have all advocated the accuracy of their methods. This paper contributes to this accuracy debate by showing how to obtain some accurate numerical results without numerical approximations, for a given investment horizon. We use a dynamic programming approach in continuous-time, and illustrate the framework with one risky and one riskless asset under a power utility. The framework is flexible enough to cover all the HARA class of utility functions.
\end{abstract}

Keywords: Dynamic portfolio choice; Long-term investing; Time aggregation; Explicit solutions; Numerical solutions.

JEL Classification: G11; G12.

\section{Introduction}

Starting from the seminal work of Merton (1971), many results on dynamic portfolio optimization problems have been obtained. However, it still appears to be difficult to provide accurate numerical results when there is predictability in asset returns, i.e. when investment opportunities are time-varying. A large number of papers have proposed to use a VAR model to forecast returns and study their implications on long-term portfolio choice problems. As a result, the academic literature has followed two main lines. The first one relies on mathematical tools, and then establishes some theoretical explicit solutions (see among others Kim and Omberg (1996), Liu (2007) and references therein). Such solutions exist only in continuous-time. To provide accurate numerical values, investor must solve a quite complicated issue of time aggregation (Bergstrom (1984) and Campbell et al. (2004)). The second line of research directly implements in discrete-time some challenging numerical methods. In fact, Barberis (2000) develops a discretization state space method that serves as a benchmark. Brandt et al. (2005), Van Binsbergen and Brandt (2007), Garlappi and Skoulakis (2009) among others use some sophisticated backward induction techniques and evaluate the accuracy of their results by comparing them to the discretization state space benchmark. Nevertheless, in a recent paper, Cong and Oosterlee (2015) implement an improved version of the method of Brandt et al. (2005), and then compare the resulting numerical values to a benchmark based on

\footnotetext{
*Érudite, Université Paris-Est, and TEPP, F.Legendre@u-pec.fr.

ॠÉrudite, Université Paris-Est, Djibril.Togola@u-pec.fr.
} 
the Fourier cosine series expansion. Using a theoretical model, we show how to analytically obtain similar numerical values.

Some approximation numerical procedures have appeared to be inconsistent. In fact, Detemple et al. (2005) find that Detemple et al. (2003) procedure is more accurate and more faster than that of Brandt et al. (2005). Van Binsbergen and Brandt (2007) using regression procedure to approximate the expectation component of value function claim that the portfolio weight iteration (which was previously developed by Brandt et al. (2005)) is more accurate than that of the value function. Garlappi and Skoulakis (2009) challenge this result by showing that certainty equivalent transformation of value function leads to much more accurate numerical results when the expectation of the value function is approximated by Gauss-Hermite quadrature with six nodes. Garlappi and Skoulakis (2011) provide a general discussion on approximations accuracy in discrete-time. However, all discrete numerical procedures approximate directly or indirectly a highly non-linear value function and cannot explicitly separate the so-called hedging demand from the so-called myopic demand. The continuous-time model considered in this paper does this separation and provides some very accurate numerical results since it is based on a well-documented explicit solution.

Boyle et al. (2008), Detemple et al. (2005), Detemple et al. (2003), Cvitanić et al. (2003) among others work in continuous-time, and they implement some numerical methods. They perform some pure simulation techniques to derive optimal portfolio weights. In fact, these authors achieve a transformation of portfolio weight as a fraction of instantaneous standard deviation of wealth or obtain solutions under Malliavin calculus, and then they carry different kinds of Monte Carlo simulations to provide numerical values. Unfortunately, these prominent techniques are intractable with the assumption of incomplete markets. We propose a direct approach derived from analytical formula with a realistic assumption of incomplete markets that respect a parsimonious VAR process for risky asset returns.

The framework we use is similar to that of Campbell et al. (2004), except we deal with horizon which does not need to be necessarily infinite, and we define the continuous state variable as the market price of risk (also called Sharpe ratio) rather than the risk premium. We focus on the clear link between the continuous state variable (market price of risk) and the discrete state variable (log dividend-price ratio). This leads to some comprehensive expressions, which are very fast to be implemented. Campbell et al. (2004) work with an approximate analytical solution for an investor with an infinite horizon and recursive preferences. In this context, they provide evidence that there should exist minor discrepancies between results under discrete vs. continuous-time models. Accordingly, numerical results we derive from continuous-time are indirectly comparable to those of Garlappi and Skoulakis (2009). We show that, for large degrees of risk aversion and/or small horizons, when the state variable is close to its unconditional mean, the two numerical results are quite similar. Otherwise, results under our explicit solution in continuous-time exhibit some discrepancies with Garlappi and Skoulakis (2009) when risk aversion decreases and/or time horizon increases. We argue that this is due to the large sensitivity of total demand to the continuous-time state variable (Sharpe ratio) or equivalently to the discrete-time state variable (log dividend-price ratio).

The paper is organized as follows; Section 1 exposes the way we map the continuous-time investment opportunity set and the discrete-time one; Section 2 gives some insights into the explicit solution for a long-term investor with CRRA preferences; Section 3 illustrates some numerical results based on the econometric model in Brandt et al. (2005) for comparison purposes, and then discusses accuracy.

\section{Investment opportunity sets}

First, we propose studying the model in a continuous-time framework, and then in a discrete-time framework to assess the impact of the predictable component in stock returns. Next, we present the way we recover continuous-time parameters that are consistent with discrete-time VAR estimates. 


\subsection{Opportunity set in continuous-time}

We start by assuming that two assets are available for the investor. On the one hand, there exists a riskless asset that pays back a constant real interest rate $r$

$$
\frac{\mathrm{d} P_{t}^{f}}{P_{t}^{f}}=r \mathrm{~d} t,
$$

where $P_{t}^{f}$ denotes the real price of this asset at time $t$; on the other hand, there is a risky asset whose real price $P_{t}$ satisfies the following diffusion process

$$
\frac{\mathrm{d} P_{t}}{P_{t}}=\mu_{t} \mathrm{~d} t+\sigma \mathrm{d} B_{t}^{p},
$$

where $B_{t}^{p}$ denotes a one-dimensional Brownian motion with zero drift and unit variance rate. The drift rate $\mu_{t}$ follows a diffusion process as well. It is supposed to be time-varying and state variable dependent. The volatility of the risky asset is assumed to be constant. This is not a strong assumption for a long-term investor (see Campbell and Viceira (2002)). Let $X_{t}$ denote the Sharpe ratio, i.e. the market price of risk/reward, for buying/selling one unit of risky asset.

$$
X_{t}=\frac{\mu_{t}-r}{\sigma} .
$$

Then, the Sharpe ratio is assumed to follow an usual "Ornstein-Uhenbeck" diffusion process

$$
\mathrm{d} X_{t}=\kappa\left(\theta-X_{t}\right) \mathrm{d} t+\zeta \mathrm{d} B_{t}^{x} \quad \kappa, \theta, \zeta>0,
$$

where $B_{t}^{x}$ denotes another one-dimensional Brownian motion with zero drift and unit variance rate. Parameters $\theta$ and $\kappa$ denote, respectively, the unconditional mean and the mean reverting parameter of the Sharpe ratio $X_{t}$. Parameter $\kappa$ reflects the rate by which shocks on Sharpe ratio dissipate, and then $X_{t}$ reverts towards its long-term mean $\theta$. Parameter $\zeta$ denotes the instantaneous volatility of $X_{t}$. It controls the diffusion rate of the process.

The above equations imply that the instantaneous return on stocks $\mathrm{d} P_{t} / P_{t}$ follows a diffusion process, whose drift is mean-reverting and whose innovations are correlated with those of the market price of risk itself, with the correlation coefficient $\rho$. Thus, the following system of equations holds;

$$
\begin{aligned}
\mathrm{d} P_{t} / P_{t} & =\left(\sigma X_{t}+r\right) \mathrm{d} t+\sigma \mathrm{d} B_{t}^{p}, \\
\mathrm{~d} X_{t} & =\kappa\left(\theta-X_{t}\right) \mathrm{d} t+\zeta \mathrm{d} B_{t}^{x},
\end{aligned}
$$

with $\mathrm{d} \boldsymbol{B}_{t}^{p} \mathrm{~d} \boldsymbol{B}_{t}^{x}=\rho \mathrm{d} t$. The market price of risk has an undiversifiable risk component meaning that markets are incomplete $(\rho \neq \pm 1)$. In fact, the equations (5) and (6) define a continuous VAR process. To see this, we just apply Ito's lemma to $\mathrm{d} P_{t} / P_{t}$, and we notice that $\mathrm{d} \theta=0$, so that we can work with matrices. After rearranging, the result is

$$
\begin{aligned}
\mathrm{d}\left[\begin{array}{c}
\ln P_{t}+\frac{1}{2} \sigma^{2} t-\sigma \theta t-r t \\
X_{t}-\theta
\end{array}\right]= & {\left[\begin{array}{cc}
0 & \sigma \\
0 & -\kappa
\end{array}\right]\left[\begin{array}{c}
\ln P_{t}+\frac{1}{2} \sigma^{2} t-\sigma \theta t-r t \\
X_{t}-\theta
\end{array}\right] \mathrm{d} t+} \\
& {\left[\begin{array}{cc}
\sigma & 0 \\
\zeta \rho & \zeta \sqrt{1-\rho^{2}}
\end{array}\right]\left[\begin{array}{l}
\mathrm{d} Z_{t}^{p} \\
\mathrm{~d} Z_{t}^{x}
\end{array}\right], }
\end{aligned}
$$

where $\mathrm{d} Z_{P, t}$ and $\mathrm{d} Z_{X, t}$ are two independent Brownian motion processes. This last expression is indeed a continuous VAR model. It could be rewritten in a compact form as

$$
\mathrm{d} Y_{t}=\mathrm{G} Y_{t} \mathrm{~d} t+\mathrm{Hd} Z_{t},
$$


where

$$
\mathrm{G}=\left[\begin{array}{cc}
0 & \sigma \\
0 & -\kappa
\end{array}\right] \text { and } \mathrm{H}=\left[\begin{array}{cc}
\sigma & 0 \\
\zeta \rho & \zeta \sqrt{1-\rho^{2}}
\end{array}\right]
$$

Equation (8) is suitable to discretize (Bergstrom (1984)). In fact, it leads to an issue of time aggregation, that we have to solve in order to provide accurate continuous-time parameters, when we take the predictability in discrete-time as given.

\subsection{Opportunity set in discrete-time}

The standard model in discrete-time is a restricted VAR(1) process which captures predictability of stock returns (see Barberis (2000) for instance). We focus on the example analyzed in Brandt et al. (2005) that was reused in Van Binsbergen and Brandt (2007) and in Garlappi and Skoulakis (2009). The log excess returns $\Delta \ln P_{t+1}-r^{f}$ are assumed to be predictable by the log dividend-price ratio $z_{t}$ $\left(r^{f}\right.$ denotes the risk-free rate and is equal to $6 \%$ on an annual basis). The joint dynamic relationship between these two variables are specified such that

$$
\begin{aligned}
\Delta \ln P_{t+1}-r^{f} & =a_{r}+b_{r} z_{t}+\varepsilon_{t+1}^{r}, \\
z_{t+1} & =a_{z}+b_{z} z_{t}+\varepsilon_{t+1}^{z},
\end{aligned}
$$

with

$$
\left[\begin{array}{c}
\varepsilon_{t+1}^{r} \\
\varepsilon_{t+1}^{z}
\end{array}\right] \sim \mathrm{N}\left(\left[\begin{array}{l}
0 \\
0
\end{array}\right],\left[\begin{array}{cc}
\sigma_{r}^{2} & \sigma_{r z} \\
\sigma_{r z} & \sigma_{z}^{2}
\end{array}\right]\right) .
$$

In fact, Campbell and Shiller (1988) forcefully claim that, if log returns are predictable, at least, the log dividend-price ratio should capture some of that predictability. A substantial long-standing empirical literature has documented many properties of these two regressions (see Cochrane (2008)). Brandt et al. (2005) report the following estimated values (using real data of the CRSP U.S. quarterly index from January 1986 to December 1995);

$$
b_{r}=0.060, \quad b_{z}=0.958, \quad \frac{\sigma_{r z}}{\sigma_{r} \sigma_{z}}=-0.941 .
$$

Log returns are weakly predictable; the log dividend-price ratio is highly persistent; and shocks are strongly negatively correlated. Hence, mean reverting occurs at longer horizons.

\subsection{Recovering continuous-time parameters from discrete-time VAR}

Closely following Campbell et al. (2004), we recover the parameters of the continuous-time system eqs (5)-(6) from the restricted VAR(1) eqs (10)-(11). However, Campbell et al. (2004) use the risk premium as a state variable and consider a specific infinitely lived long term investor; we explore the case of the Sharpe ratio with a finite or infinite long term investment horizon. We focus on the clear link between the continuous state variable (market price of risk) and the discrete state variable (dividend-price ratio).

The first step is to aggregate the continuous-time model over a span of time, taking point observations at evenly spaced points $\left\{t_{0}, t_{1}, \ldots, t_{n}, t_{n+1}, \ldots\right\}$, with $\Delta t=t_{n}-t_{n-1}$. Using the discretization method developed by Bergstrom (1984), we then obtain

$$
\begin{aligned}
{\left[\begin{array}{c}
\Delta \ln P_{t_{n}+\Delta t}-r \Delta t \\
X_{t_{n}+\Delta t}
\end{array}\right]=} & {\left[\begin{array}{c}
\left(\sigma \theta-\sigma^{2} / 2\right) \Delta t-\left(1-\mathrm{e}^{-\kappa \Delta t}\right) \frac{\sigma \theta}{\kappa} \\
\left(1-\mathrm{e}^{-\kappa \Delta t}\right) \theta
\end{array}\right]+} \\
& {\left[\begin{array}{cc}
1 & \left(1-\mathrm{e}^{-\kappa \Delta t}\right) \frac{\sigma}{\kappa} \\
0 & \mathrm{e}^{-\kappa \Delta t}
\end{array}\right]\left[\begin{array}{c}
\Delta \ln P_{t_{n}}-r \\
X_{t_{n}}
\end{array}\right]+\left[\begin{array}{c}
U_{t_{n}+\Delta t}^{p} \\
U_{t_{n}+\Delta t}^{x}
\end{array}\right], }
\end{aligned}
$$




\begin{tabular}{|c|c|c|c|}
\hline \multicolumn{2}{|c|}{ Discrete-time world } & \multicolumn{2}{|c|}{ Continuous-time world } \\
\hline \multicolumn{4}{|c|}{ Models } \\
\hline \multirow{2}{*}{\multicolumn{2}{|c|}{$\begin{aligned} & \text { Brandt et al. (2005) } \\
& \Delta \ln P_{t+1}-r^{f}=a_{r}+b_{r} z_{t}+\varepsilon_{t+1}^{r} \\
& z_{t+1}=a_{z}+b_{z} z_{t}+\varepsilon_{t+1}^{z} \\
& \mathrm{~V}\left(\varepsilon_{t}^{r}\right)=\sigma_{r}^{2} \\
& \mathrm{~V}\left(\varepsilon_{t}^{z}\right)=\sigma_{s}^{2} \\
& \operatorname{Cov}\left(\varepsilon_{t}^{r}, \varepsilon_{t}^{z}\right)=\sigma_{r z}\end{aligned}$}} & \multirow{2}{*}{\multicolumn{2}{|c|}{$\begin{aligned} & \text { Kim and Omberg (1996) } \\
& \mathrm{d} P_{t}^{f} / P_{t}^{f}=r \mathrm{~d} t \\
& \mathrm{~d} P_{t} / P_{t}=\left(\sigma X_{t}+r\right) \mathrm{d} t+\sigma \mathrm{d} B_{t}^{p} \\
& \mathrm{~d} X_{t}=\kappa\left(\theta-X_{t}\right) \mathrm{d} t+\zeta \mathrm{d} B_{t}^{x} \\
& \mathrm{~d} B_{t}^{p} \mathrm{~d} B_{t}^{x}=\rho \mathrm{d} t\end{aligned}$}} \\
\hline & & & \\
\hline \multicolumn{4}{|c|}{ Parameter values } \\
\hline \multicolumn{2}{|c|}{ Brandt et al. (2005) } & \multicolumn{2}{|c|}{ Our computations eqs (16)-(21) } \\
\hline & 0.015 & & \\
\hline$a_{r}$ & 0.227 & $r$ & 0.015 \\
\hline$b_{r}$ & 0.060 & & 0.111 \\
\hline$a_{z}$ & -0.155 & $\kappa$ & 0.0429 \\
\hline$b_{z}$ & 0.958 & & 0.0775 \\
\hline$\sigma_{r}^{2}$ & 0.0060 & $\zeta$ & 0.0542 \\
\hline$\sigma_{z}^{2}$ & 0.0049 & $\rho$ & -0.941 \\
\hline$\sigma_{r z}$ & -0.0051 & & \\
\hline
\end{tabular}

Table 1: Recovering of continuous-time parameters

where

$$
\left[\begin{array}{l}
U_{t_{n}+\Delta t}^{p} \\
U_{t_{n}+\Delta t}^{x}
\end{array}\right]=\int_{\tau=0}^{\Delta t}\left[\begin{array}{cc}
1 & \left(1-\mathrm{e}^{-\kappa \Delta t}\right) \frac{\sigma}{\kappa} \\
0 & \mathrm{e}^{-\kappa \Delta t}
\end{array}\right]\left[\begin{array}{cc}
\sigma & 0 \\
\zeta \rho & \zeta \sqrt{1-\rho^{2}}
\end{array}\right]\left[\begin{array}{l}
\mathrm{d} B_{t_{n}+\tau}^{p} \\
\mathrm{~d} Z_{t_{n}+\tau}^{x}
\end{array}\right],
$$

with $\mathrm{d} B_{t}^{x}=\rho \mathrm{d} B_{t}^{p}+\sqrt{1-\rho^{2}} \mathrm{~d} Z_{t}^{x}$ where $B_{t}^{p}$ and $Z_{t}^{x}$ are two independent Brownian terms. Appendix A.1 derives the equations (13) and (14).

The second step considers a matrix form of the estimates of the discrete-time VAR system, eqs (10)-(11)

$$
\left[\begin{array}{c}
\Delta \ln P_{t+1}-r^{f} \\
z_{t+1}
\end{array}\right]=\left[\begin{array}{c}
a_{r} \\
a_{z}
\end{array}\right]+\left[\begin{array}{ll}
0 & b_{r} \\
0 & b_{z}
\end{array}\right]\left[\begin{array}{c}
\Delta \ln P_{t}-r^{f} \\
z_{t}
\end{array}\right]+\left[\begin{array}{c}
\varepsilon_{t+1}^{r} \\
\varepsilon_{t+1}^{z}
\end{array}\right] .
$$

Then, we apply a linear transformation for the process $X_{t}$ in (13). This allows us to relate the parameters of the transformed systems in (13) and (15). Hence, when we normalize the time span $\Delta t=1$, since everything is in quarterly frequency, we get (for $b_{z}, b_{r}>0$ )

$$
\begin{aligned}
r & =r^{f}, \\
\theta & =\frac{a_{z} b_{r}}{\sigma_{r}\left(1-b_{z}\right)}+\frac{a_{r}+\sigma_{r}^{2} / 2}{\sigma_{r}}, \\
\kappa & =-\ln \left(b_{z}\right), \\
\sigma & =\sigma_{r} \\
\zeta & =b_{r} \frac{\sigma_{z}}{\sigma_{r}} \\
\rho & =\frac{\sigma_{r z}}{\sigma_{r} \sigma_{z}} .
\end{aligned}
$$

Appendix A.2 proves these results. Table 1 illustrates the values of the parameters of the continuoustime VAR implied by the Brandt et al. (2005) estimates. 


\section{Portfolio choice problem in continuous-time with CRRA preferences}

We can now solve the portfolio choice problem of the investor with a long-term horizon, who is confronted with the investment opportunity set described in section 1.1. We rely on recent advances in Honda and Kamimura (2011), who use the verification theorem to show that the explicit solution provided in continuous-time is, in fact, an optimal solution, especially for risk aversion greater than one.

Let's consider an investor with a positive initial wealth $W_{t_{0}}>0$, who could only invest in two assets, for instance, a riskless short-term bond and a well-diversified stock index. Markets are incomplete. Furthermore, the investor can undertake continuous trading; he has no labor incomes and only cares about the utility of terminal wealth $W_{T}>0$, where $T$ is the finite or infinite planning horizon. The dynamics of price changes are described by (1) and (5)-(6). If $\alpha_{t}$ defines the share of wealth invested in stocks, the instantaneous wealth would be given by $\mathrm{d} W_{t} / W_{t}=$ $\alpha_{t} \mathrm{~d} P_{t} / P_{t}+\left(1-\alpha_{t}\right) \mathrm{d} P_{t}^{f} / P_{t}^{f}$. Properly substituting the dynamics of $\mathrm{d} P_{t} / P_{t}$ and $\mathrm{d} P_{t}^{f} / P_{t}^{f}$, wealth dynamics (also called the budget constraint) become

$$
\mathrm{d} W_{t}=\left(\alpha_{t} \sigma X_{t}+r\right) W_{t} \mathrm{~d} t+\alpha_{t} \sigma W_{t} \mathrm{~d} B_{t}^{p} .
$$

Notice that the wealth process reflects uncertainty in instantaneous returns $\left(\mathrm{d} \boldsymbol{B}_{t}^{p}\right.$ term) and in the state variable (term in $X_{t}$ ). Given this formalization of the wealth process, at time $t_{0}$, the investor's optimization problem can then be expressed as

$$
\max _{\alpha_{t}} \mathrm{E}_{t_{0}} u\left(W_{T}\right) \quad \text { s.t. (22), } \quad W_{t_{0}} \text { fixed. }
$$

where $\mathrm{E}_{t_{0}}$ denotes the operator of conditional expectation at date $t_{0}$, starting the process at $t=t_{0}$, and $u(\cdot)$ the utility function defined over terminal wealth. Since only the utility of terminal wealth does matter, no time discount parameter would be required. Let $J\left(W_{t_{0}}, X_{t_{0}}, t_{0}\right)$ define the value of the investor's problem at time $t_{0}$

$$
J\left(W_{t_{0}}, X_{t_{0}}, t_{0}\right)=\max _{\alpha_{t}} \mathrm{E}_{t_{0}} u\left(W_{T}\right) .
$$

The Bellman principle of optimality generalizes this problem to every time $t$, so that

$$
J\left(W_{t}, X_{t}, t\right)=\max _{\alpha_{t}} \mathrm{E}_{t} J\left(W_{t}+\mathrm{d} W_{t}, X_{t}+\mathrm{d} X_{t}, t+\mathrm{d} t\right) .
$$

The Bellman equation in (25) emphasizes on the idea that current optimal decision is made by assuming that we will make optimal decisions in all future periods. Hence, we take into account that our current decision provides the maximum expected utility we can reach respecting all the future optimal decisions. The expectation $\mathrm{E}_{t} J\left(W_{t}+\mathrm{d} W_{t}, X_{t}+\mathrm{d} X_{t}, t+\mathrm{d} t\right)$ depends on $\alpha_{t}$. Applying Ito's lemma to the Bellman equation, we obtain

$$
\begin{gathered}
0=\max _{\alpha_{t}}\left(\frac{\partial J}{\partial W_{t}}\left(\alpha_{t} \sigma X_{t}+r\right) W_{t}+\frac{\partial J}{\partial t}+\frac{\partial J}{\partial X_{t}} \kappa\left(\theta-X_{t}\right)+\frac{1}{2} \frac{\partial^{2} J}{\partial^{2} W_{t}} \sigma^{2} \alpha_{t}^{2} W_{t}^{2}+\right. \\
\left.\frac{1}{2} \frac{\partial^{2} J}{\partial^{2} X_{t}} \zeta^{2}+\frac{\partial^{2} J}{\partial W_{t} \partial X_{t}} \sigma \alpha_{t} \zeta \rho W_{t}\right) .
\end{gathered}
$$

The first order condition of equation (26) with respect to $\alpha_{t}$ implies that

$$
\alpha_{t}^{\star}=-\frac{\partial J / \partial W_{t}}{\partial^{2} J / \partial^{2} W_{t}} \frac{1}{W_{t}} \frac{X_{t}}{\sigma}-\frac{\partial^{2} J /\left(\partial W_{t} \partial X_{t}\right)}{\partial^{2} J / \partial^{2} W_{t}} \frac{1}{W_{t}} \frac{\zeta}{\sigma} \rho .
$$

Merton (1971) was the first to propose such additive decomposition between a myopic demand (first term) and a hedging demand (second term) of the optimal allocation to stocks on the right hand side 
of (27). As special cases, there is no hedging demand when the opportunity set is nonstochastic $(\zeta=0)$, or when the opportunity set is uncorrelated with asset returns $(\rho=0)$. Otherwise, to solve the problem, we need to explicitly define the function $J(\cdot)$.

The first conjecture is

$$
J\left(W_{t}, X_{t}, t\right)=u\left(W_{t}\right)\left[f\left(X_{t}, t\right)\right]^{\gamma},
$$

where $f(\cdot)$ is an auxiliary function with the terminal condition $f\left(X_{T}, T\right)=1$. We consider a CRRA preferences $u\left(W_{t}\right)=W_{t}^{1-\gamma} /(1-\gamma)$, where $\gamma$ is the coefficient of relative risk aversion. Thus, the hedging demand in (27) could straightforward be expressed as

$$
\frac{\partial f / \partial X_{t}}{f} \frac{\zeta}{\sigma} \rho=\frac{\partial \ln f}{\partial X_{t}} \frac{\zeta}{\sigma} \rho
$$

Then, under CRRA preferences, the optimal allocation to stocks becomes

$$
\alpha_{t}^{\star}=\frac{1}{\gamma} \frac{X_{t}}{\sigma}+\frac{\partial \ln f}{\partial X_{t}} \frac{\zeta}{\sigma} \rho
$$

So, the Bellman equation (26) could be rewritten as

$$
0=\frac{f_{t}^{\prime}}{f}+\frac{1-\gamma}{\gamma} r+\frac{1-\gamma}{2 \gamma^{2}} X_{t}^{2}+\frac{f_{x}^{\prime}}{f}\left[\frac{1-\gamma}{\gamma} \zeta X_{t} \rho+\kappa\left(\theta-X_{t}\right)\right]+\frac{f_{x x}^{\prime \prime}}{f} \frac{\zeta^{2}}{2}+\left(\frac{f_{x}^{\prime}}{f}\right)^{2} \frac{1-\gamma}{2} \zeta^{2}\left(\rho^{2}-1\right)
$$

where we use intuitive notations for the derivatives of the function $f(\cdot)$. Equation (30) is a partial differential equation which admits analytical solutions especially if utility is logarithmic $(\gamma=1$ by l'Hopital's rule) or if markets are complete $(\rho= \pm 1)$.

The second conjecture is to assume

$$
f\left(X_{t}, t\right)=\exp \left(C_{0}(t)+C_{1}(t) X_{t}+\frac{1}{2} C_{2}(t) X_{t}^{2}\right),
$$

where $C_{0}(t), C_{1}(t)$ and $C_{2}(t)$ are some undetermined time varying coefficients (with $C_{0}(T)=$ $C_{1}(T)=C_{2}(T)=0$ ). Under this conjecture, using equation (29), the intertemporal optimal allocation to stocks is

$$
\alpha_{t}^{\star}=\frac{1}{\gamma} \frac{X_{t}}{\sigma}+\left[C_{1}(t)+C_{2}(t) X_{t}\right] \frac{\zeta}{\sigma} \rho .
$$

We only need to recover $C_{1}(t)$ and $C_{2}(t)$ coefficients.

The conjectures in (28) and (31) are due to Kim and Omberg (1996) followed by Liu (2007) among others. More recently, Honda and Kamimura (2011) analytically show that the explicit solution derived from the Bellman equation is in fact, an optimal solution to the problem of the long-term investor with CRRA preferences and risk aversion greater than one.

Let us substitute the second conjecture into the equation (30)

$$
\begin{aligned}
0=\left(\frac{\mathrm{d} C_{2}}{\mathrm{~d} t}+a C_{2}^{2}+b C_{2}+c\right) X_{t}^{2}+ & \left(\frac{\mathrm{d} C_{1}}{\mathrm{~d} t}+\frac{b}{2} C_{1}+\kappa \theta C_{2}+a C_{1} C_{2}\right) X_{t}+ \\
& \left(\frac{\mathrm{d} C_{0}}{\mathrm{~d} t}+\frac{1-\gamma}{\gamma} r+\kappa \theta C_{1}+\frac{\zeta^{2}}{2} C_{2}+\frac{a}{2} C_{1}^{2}\right),
\end{aligned}
$$

where $a=\left[1+(1-\gamma)\left(\rho^{2}-1\right)\right] \zeta^{2}, b=2[\zeta \rho(1-\gamma) / \gamma-\kappa]$ and $c=(1-\gamma) / \gamma^{2}$. As whatever the value of $X_{t}$, the equation (33) must hold, all terms within parentheses in (33) are simultaneously set to zero in order to solve the equation for $C_{0}(\cdot), C_{1}(\cdot)$, and $C_{2}(\cdot)$. Defining the discriminant $D$

$$
D=b^{2}-4 a c,
$$




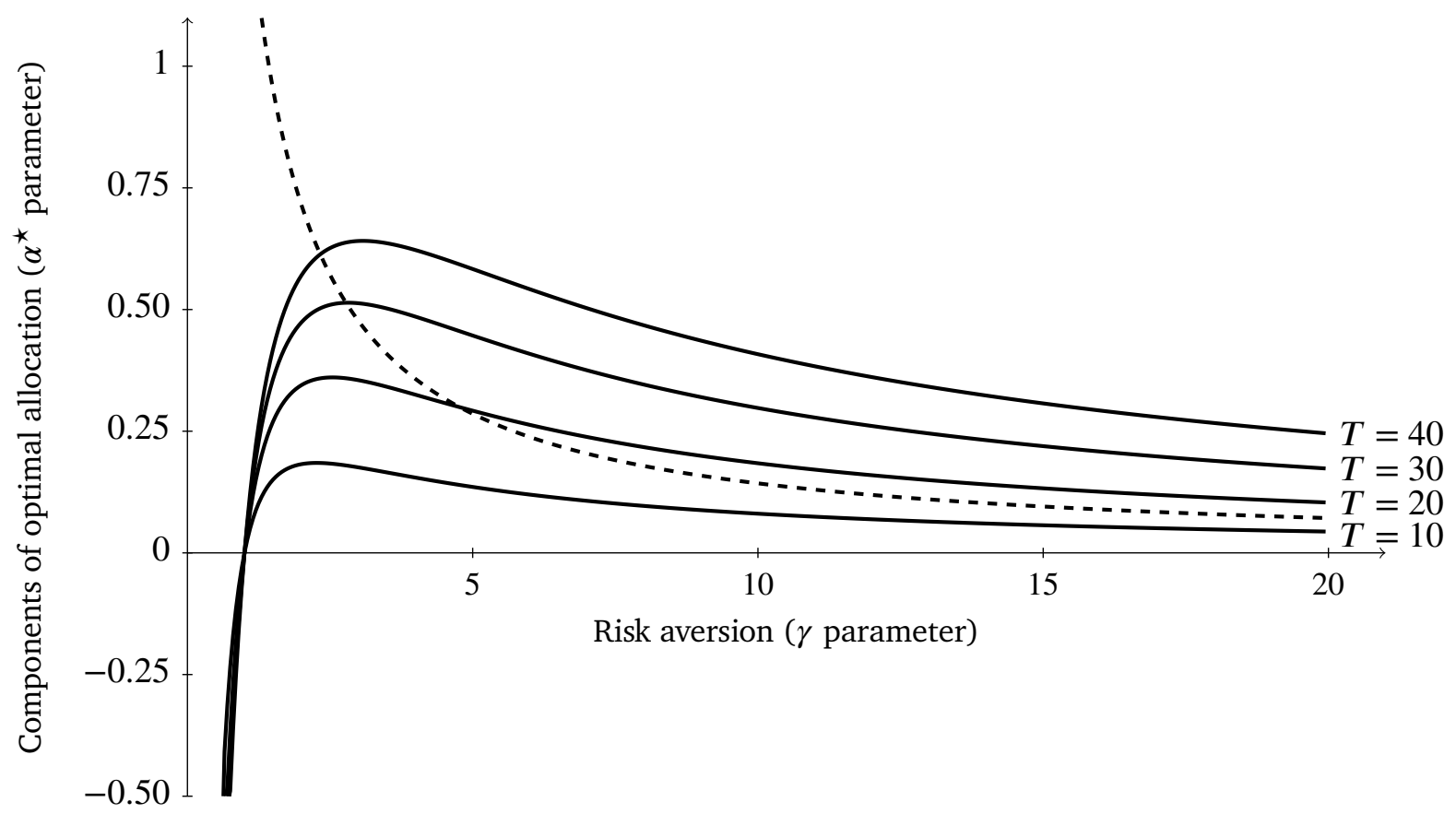

Figure 1: Myopic (dashed line) and hedging (solid line) demands as function of risk aversion for $X_{t_{0}}=\theta$

one can verify that if $\gamma>1$, then $D>0$. Thus, the two solutions of interest would be given by

$$
\begin{aligned}
& C_{2}(t)=\frac{2 c\left(1-\mathrm{e}^{-\delta(T-t)}\right)}{2 \delta-(b+\delta)\left(1-\mathrm{e}^{-\delta(T-t)}\right)} \\
& C_{1}(t)=\frac{4 c \kappa \theta}{\delta} \frac{\left(1-\mathrm{e}^{-\delta(T-t) / 2}\right)^{2}}{2 \delta-(b+\delta)\left(1-\mathrm{e}^{-\delta(T-t)}\right)},
\end{aligned}
$$

where $\delta$ denotes $\sqrt{D}$. In a context of HARA utility functions, Kim and Omberg (1996) call the implied portfolio, the normal solution, and they discuss about some alternative solutions those are beyond the scope of this paper. The appendix A.3 provides details on the derivation of (34) and (35). It is easy to see that, at each point in time, $C_{1}(\cdot)$ is proportional to $C_{2}(\cdot)$, and $C_{1}(\cdot)$ takes the sign of $C_{2}(\cdot)$. Thus, for $\gamma>1$, then $C_{1}$ and $C_{2}$ are always negative. As a result, since $\rho<0$, the hedging demand is always positive when the preferences are not logarithmic (more precisely for $\gamma>1$ ) and the market price of risk is positive. Such consistent theoretical result could be used to provide numerical values.

\section{Numerical results}

As mentioned above, for the purposes of comparison, as in Garlappi and Skoulakis (2009), we illustrate our approach using the well documented econometric model in Brandt et al. (2005). Table 1 collects the continuous-time parameters recovered from this example. We also collect in table 3 a sample of numerical results in Garlappi and Skoulakis (2009) by means of a sophisticated numerical method.

Figure 1 and table 2 help to understand the long-term investor problem. For $\gamma=1$ i.e., the case of logarithmic utility, no hedging demand is required. In this case, the dynamic portfolio choice is reduced to the static one, whatever the time horizon. Otherwise, for $\gamma>1$ and horizons longer 


\begin{tabular}{|c|c|c|c|c|c|c|c|c|c|c|c|}
\hline \multirow[b]{2}{*}{$T$} & & \multicolumn{5}{|l|}{$\gamma=5$} & \multicolumn{5}{|c|}{$\gamma=15$} \\
\hline & & $X_{(10)}$ & $X_{(30)}$ & $X_{(50)}$ & $X_{(70)}$ & $X_{(90)}$ & $X_{(10)}$ & $X_{(30)}$ & $X_{(50)}$ & $X_{(70)}$ & $X_{(90)}$ \\
\hline \multirow[t]{2}{*}{10} & MD & -34.0 & 3.0 & 28.6 & 54.2 & 91.1 & -11.3 & 1.0 & 9.5 & 18.1 & 30.4 \\
\hline & HD & -10.9 & 3.5 & 13.5 & 23.6 & 38.0 & -4.6 & 1.5 & 5.7 & 9.9 & 15.9 \\
\hline \multirow[t]{2}{*}{20} & MD & -34.0 & 3.0 & 28.6 & 54.2 & 91.1 & -11.3 & 1.0 & 9.5 & 18.1 & 30.4 \\
\hline & HD & -15.9 & 10.8 & 29.2 & 47.7 & 74.3 & -7.2 & 4.9 & 13.3 & 21.6 & 33.7 \\
\hline \multirow[t]{2}{*}{30} & MD & -34.0 & 3.0 & 28.6 & 54.2 & 91.1 & -11.3 & 1.0 & 9.5 & 18.1 & 30.4 \\
\hline & HD & -16.0 & 19.8 & 44.7 & 69.5 & 105.3 & -7.7 & 9.8 & 21.9 & 34.1 & 51.6 \\
\hline \multirow[t]{2}{*}{40} & MD & -34.0 & 3.0 & 28.6 & 54.2 & 91.1 & -11.3 & 1.0 & 9.5 & 18.1 & 30.4 \\
\hline & HD & -13.2 & 29.1 & 58.3 & 87.6 & 129.8 & -6.5 & 15.5 & 30.7 & 46.0 & 68.0 \\
\hline
\end{tabular}

For each risk aversion $\gamma$, the first line reports the myopic demand (MD) and the second line the hedging demand (HD) without short sale constraints. We present the results for 5 different initial values of the Sharpe ratio $X$. Each value corresponds to the $p$-th percentile of the unconditional distribution of $X$, defined by equation (59) and denoted by $X_{(p)}$, where $p$ takes values 10, 30, 50, 70, and 90 (then $X_{(50)}=\theta$ ).

Table 2: Myopic and hedging demands for investment horizon of $T$ quarters (\%)

than one, under CRRA preferences and mean reverting returns, the agent should have a positive hedging demand to prevent adverse changes in investment opportunities (Merton, 1971). However, for $\gamma \rightarrow+\infty$, more specifically for a very conservative agent, stocks are not attractive. Thus, he would not invest in stocks, since the total demand (sum of myopic and hedging demands) converges towards zero. Our results recover all these important basic features.

The total demand is sensitive to risk aversion. Results from previous studies imply that myopic and hedging demands are more sensitive to small values of risk aversion. We confirm this result and argue that the sensitivity of hedging demand to a state variable is maximal near the critical point $\gamma \approx 2$. Equation (32) and figure 1 illustrate this point. To quantitatively highlight this result, just evaluate the derivative of $\alpha^{\star}$ with respect to $X$.

Table 2 reports the evidence that both myopic and hedging demands are sensitive to initial values of the Sharpe ratio. Each element of these two components of optimal allocation monotonically increases with the percentile of the Sharpe ratio unconditional distribution. Thus, the total demand exhibits the same behavior. This is consistent with Garlappi and Skoulakis (2009) and Campbell et al. (2004) among others. In fact, high Sharpe ratio or equivalently high risk premium relative to volatility, signals better investment opportunities. Therefore, optimal fraction to allocate in stocks should increase.

Myopic demand is independent from time horizon, while hedging demand increases nonlinearly with time horizon. However, table 2 quantitative figures suggest that this relation is almost linear, but small changes in horizon induce substantial hedging demand. Horizon effect is important and quite linear for a given percentile of the state variable unconditional distribution. All changes in total demand for fixed risk aversion and state variable are due to changes in horizon and are large for small risk aversion (see figure 2).

With regard to optimal allocation, the horizon effect on hedging demand is important, because it widely dominates at longer horizons. In fact, when the horizon is greater than 20 quarters, hedging demand is always greater than myopic demand, when the Sharpe ratio initial values do lie between 30 and 70 percentiles (table 2).

Finally, let's take the case of the common assumption of no-borrowing and no-short-sale constraints. Thus, in table 3 , we restrict all portfolio weights between 0 and 1 . It can be noticed that we generally obtain values fairly close to those of Garlappi and Skoulakis (2009), while frameworks are not the same. Garlappi and Skoulakis (2009) work in discrete-time and initial values of their state variable are drawn for the unconditional distribution of quarterly dividend-price ratios. They use a 

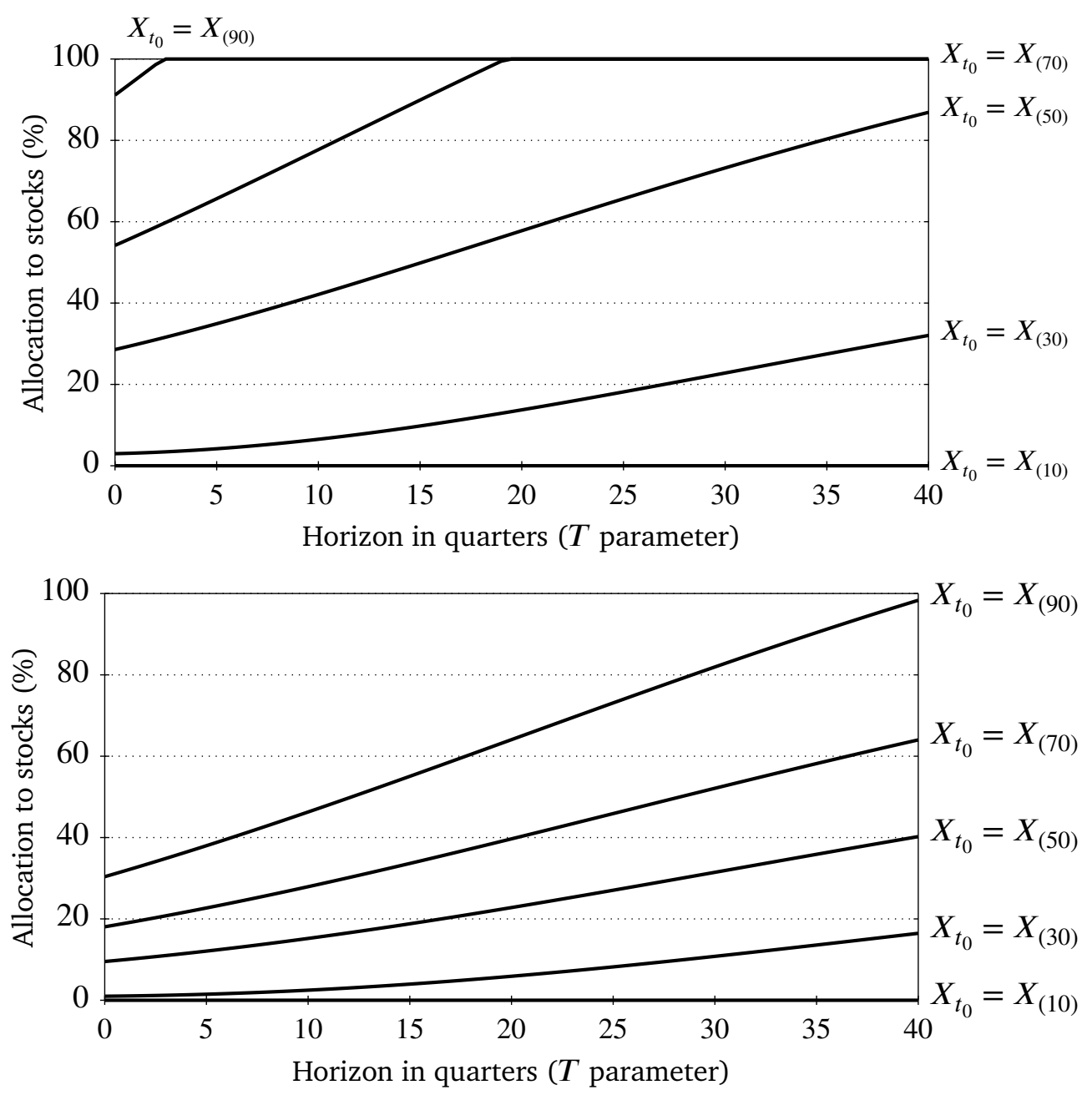

Figure 2: Optimal allocation to stocks as function of horizon for $\gamma=5$ (first panel) and for $\gamma=15$ (second panel) for 5 different initial values of the Sharpe ratio $X$ (as in table 2 or 3 ) 


\begin{tabular}{|c|c|c|c|c|c|c|c|c|c|c|c|}
\hline \multirow[b]{2}{*}{$T$} & & \multicolumn{5}{|l|}{$\gamma=5$} & \multicolumn{5}{|c|}{$\gamma=15$} \\
\hline & & $X_{(10)}$ & $X_{(30)}$ & $X_{(50)}$ & $X_{(70)}$ & $X_{(90)}$ & $X_{(10)}$ & $X_{(30)}$ & $X_{(50)}$ & $X_{(70)}$ & $X_{(90)}$ \\
\hline \multirow[t]{3}{*}{10} & LT & 0.0 & 6.5 & 42.1 & 77.7 & 100.0 & 0.0 & 2.5 & 15.2 & 27.9 & 46.3 \\
\hline & GS & 0.0 & 13.3 & 43.2 & 73.1 & 100.0 & 0.0 & 4.3 & 15.4 & 27.0 & 44.7 \\
\hline & $\Delta$ & 0.0 & -6.8 & -1.1 & 4.6 & 0.0 & 0.0 & -1.8 & -0.2 & 0.9 & 1.6 \\
\hline \multirow[t]{3}{*}{20} & LT & 0.0 & 13.7 & 57.8 & 100.0 & 100.0 & 0.0 & 5.9 & 22.8 & 39.7 & 64.1 \\
\hline & GS & 0.0 & 24.4 & 57.2 & 89.7 & 100.0 & 0.0 & 10.7 & 25.1 & 40.4 & 63.2 \\
\hline & $\Delta$ & 0.0 & -10.7 & 0.6 & 10.3 & 0.0 & 0.0 & -4.8 & -2.3 & -0.7 & 0.9 \\
\hline \multirow[t]{3}{*}{30} & LT & 0.0 & 22.8 & 73.2 & 100.0 & 100.0 & 0.0 & 10.8 & 31.5 & 52.1 & 81.9 \\
\hline & GS & 0.0 & 32.8 & 68.4 & 100.0 & 100.0 & 0.0 & 17.5 & 35.2 & 54.0 & 80.7 \\
\hline & $\Delta$ & 0.0 & -10.0 & 4.8 & 0.0 & 0.0 & 0.0 & -6.7 & -3.7 & -1.9 & 1.2 \\
\hline \multirow[t]{3}{*}{40} & LT & 0.0 & 32.0 & 86.9 & 100.0 & 100.0 & 0.0 & 16.5 & 40.2 & 64.0 & 98.3 \\
\hline & GS & 0.0 & 38.8 & 77.6 & 100.0 & 100.0 & 0.0 & 24.1 & 44.5 & 65.7 & 94.6 \\
\hline & $\Delta$ & 0.0 & -6.8 & 9.3 & 0.0 & 0.0 & 0.0 & -7.6 & -4.3 & -1.7 & 3.7 \\
\hline
\end{tabular}

With the restriction of no levered positions, for each risk aversion $\gamma$, the first line reports our results (LT optimal allocation to stocks in continuous-time), the second line the Garlappi and Skoulakis (2009) results (GS - optimal allocation to stocks in discrete-time), and the third line reports the difference between them. We present the results for 5 different initial values of the Sharpe ratio $X$, calibrated using the same estimates, involving dividend-price ratio as in GS. Each value corresponds to the $p$-th percentile of the unconditional distribution of $X$, defined by equation (59) and denoted by $X_{(p)}$, where $p$ takes values 10, 30, 50, 70, and 90.

Table 3: Optimal allocation to stocks for investment horizon of $T$ quarters (\%)

sophisticated numerical optimization technique. We work in continuous-time (no numerical optimization) and our initial values are computed using the unconditional distribution of the continuous Sharpe ratio, that we discretize in point observations and recover using the same quarterly dividendprice ratios. However, a closer inspection of table 3 figures points out that the optimal allocation to stocks is more sensitive to the state variable and the time horizon than the sensitivity obtained by Garlappi and Skoulakis (2009). We ran some numerical simulations, within the discrete-time framework, to evaluate our results in order to find the causes of the discrepancies between the two frameworks. We were unable to qualitatively or quantitatively invalidate our results.

To test our results, we ran some forward pure simulations in discrete-time. More precisely, for instance, we illustrate the critical case where the initial value of the Sharpe ratio is the 30-th percentile $\left(X_{t}=X_{(30)}\right)$, the relative risk aversion is equal to $5(\gamma=5)$, and the planning horizon is set to 10 ( $T=10$ quarters). Within this configuration, when we get an initial optimal allocation to stocks of 0.065 , Garlappi and Skoulakis (2009) obtain twice as many (0.133, see table 3$)$. That discrepancy is significant. Thus, we first build a sample of size 100000 for $z_{t+1}, z_{t+2}, \ldots, z_{t+10}$ and $\Delta \ln P_{t+1}, \Delta \ln P_{t+2}, \ldots, \Delta \ln P_{t+10}$ using the restricted VAR(1) eqs (10)-(11). We choose the grid $g=\{0.05,0.10,0.15,0.20,0.25\}$ for trial allocations to stocks, to overlay both our and Garlappi and Skoulakis (2009)'s solutions. Then, for each path in the sample, the value of terminal wealth is computed from the Cartesian product $g \times g \times \cdots \times g$ of all possible strategies. The computational burden is very high, as we evaluate $5^{10}=9765625$ strategies. Figure 3 shows that the forward path in discrete-time (no numerical optimization) is close to the path of our explicit solution, particularly at the critical starting point, the 30-th percentile of the state variable for small risk aversion $(\gamma=5)$. Furthermore, the resulting portfolio weight is 0.05 , which is very close to 0.065 , the value we find.

When we take predictability of returns as given, even if the market price of risk is unobservable, one can definitely compute its first and second moments (see appendix A.2) at every time $t$, and then derive explicit tractable relations for all the continuous-time parameters. We use such a framework to provide numerical results we were unable to reject. We consider CRRA preferences; however, when the discrete VAR model considered in this paper is relevant, one could provide similar numerical 


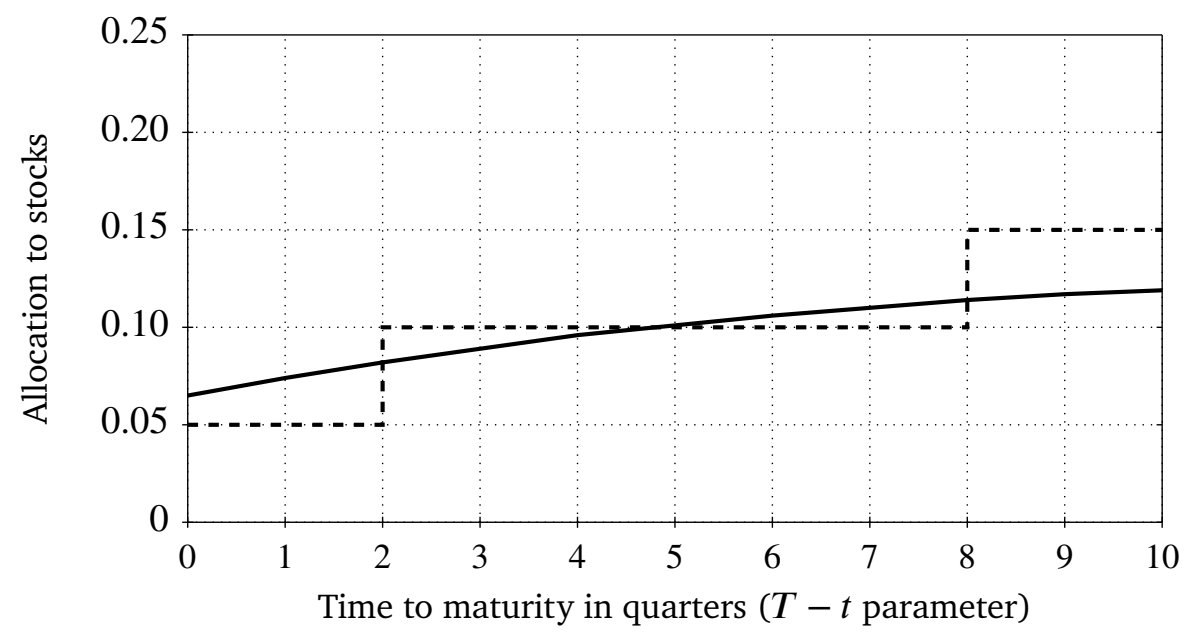

Figure 3: Path of optimal allocation to stocks for $\gamma=5, X_{0}=X_{(30)}$, and $T=10$ obtained by explicit solution (solid line) and by simulation and trial $\{0.05,0.10,0.15,0.20,0.25\}$ grid (dashed line)

results for all the HARA class of utility functions based on Kim and Omberg (1996) theoretical results, since the equations (16)-(21) and (59) hold.

\section{Conclusion}

In this paper, we analytically address the issue of numerical accuracy of a dynamic portfolio choice problem, with CRRA preferences, for a given time horizon. Based on some theoretical advances on the subject, we demonstrate that numerical values ${ }^{1}$ provided by explicit solutions are very accurate. Intensive simulations support this conclusion.

Previous numerical approximation techniques that deal with the problem we consider, are subject to some numerical errors. Therefore, they do not always provide accurate numerical results. Using an explicit solution, we show how to obtain both conditional and unconditional distributions of the market price of risk, which are consistent with an econometric model; then, we derive tractable analytical expressions for all the continuous-time parameters. Such analytical expressions are consistent with all the HARA class of utility functions (see Kim and Omberg (1996)). Numerical results with CRRA preferences highlight that 1 - portfolio weights are more sensitive to changes in risk aversion than sensitivities reported in the literature; 2- the sensitivity of total demand to risk aversion is not uniform along the unconditional distribution of state variables; 3- hedging demand dominates at longer horizons, and it is very sensitive to state variables, especially when risk aversion decreases and/or time horizon increases. These findings could partially explain the low accuracy of some discrete numerical methods, especially along the tails of the unconditional distribution of a state variable.

\section{References}

Barberis, N. (2000). Investing for the long run when returns are predictable. Journal of Finance, 55(1):225-264.

Bergstrom, A. R. (1984). Continuous time stochastic models and issues of aggregation over time.

\footnotetext{
${ }^{1}$ Complete MATLAB and $\mathrm{C}++$ codes are available by the authors upon request.
} 
In Griliches, Z. and Intriligator, M. D., editors, Handbook of Econometrics, volume 2, chapter 20, pages 1145-1212. Elsevier, 1 edition.

van Binsbergen, J. H. and Brandt, M. W. (2007). Solving dynamic portfolio choice problems by recursing on optimized portfolio weights or on the value function? Computational Economics, 29(3-4):355-367.

Boyle, P., Imai, J., and Tan, K. S. (2008). Computation of optimal portfolios using simulation-based dimension reduction. Insurance: Mathematics and Economics, 43(3):327 - 338.

Brandt, M. W., Goyal, A., Santa-Clara, P., and Stroud, J. R. (2005). A simulation approach to dynamic portfolio choice with an application to learning about return predictability. Review of Financial Studies, 18(3):831-873.

Campbell, J. Y., Chacko, G., Rodriguez, J., and Viceira, L. M. (2004). Strategic asset allocation in a continuous-time VAR model. Journal of Economic Dynamics and Control, 28(11):2195-2214.

Campbell, J. Y. and Shiller, R. J. (1988). Stock prices, earnings, and expected dividends. The Journal of Finance, 43(3):661-676.

Campbell, J. Y. and Viceira, L. M. (2002). Strategic Asset Allocation: Portfolio Choice for Long-Term Investors. Oxford University Press.

Cochrane, J. H. (2008). The dog that did not bark: A defense of return predictability. Review of Financial Studies, 21(4):1533-1575.

Cong, F. and Oosterlee, C. W. (2015). Accurate and robust numerical methods for the dynamic portfolio management problem. Available at SSRN 2563049.

Cvitanić, J., Goukasian, L., and Zapatero, F. (2003). Monte Carlo computation of optimal portfolios in complete markets. Journal of Economic Dynamics and Control, 27(6):971 - 986.

Detemple, J., Garcia, R., and Rindisbacher, M. (2005). Intertemporal asset allocation: A comparison of methods. Journal of Banking and Finance, 29(11):2821 - 2848.

Detemple, J. B., Garcia, R., and Rindisbacher, M. (2003). A Monte Carlo method for optimal portfolios. The Journal of Finance, 58(1):401-446.

Garlappi, L. and Skoulakis, G. (2009). Numerical solutions to dynamic portfolio problems: The case for value function iteration using taylor approximation. Computational Economics, 33(2):193-207.

Garlappi, L. and Skoulakis, G. (2011). Taylor series approximations to expected utility and optimal portfolio choice. Mathematics and Financial Economics, 5(2):121-156.

Gradshteyn, I. and Ryzhik, I. (2007). Table of integrals, series, and products. Translated from the Russian. 7th ed. Amsterdam: Elsevier/Academic Press, 7th ed. edition.

Hamilton, J. D. (1994). Time Series Analysis. Princeton University Press.

Honda, T. and Kamimura, S. (2011). On the verification theorem of dynamic portfolio-consumption problems with stochastic market price of risk. Asia-Pacific Financial Markets, 18(2):151-166.

Kim, T. and Omberg, E. (1996). Dynamic nonmyopic portfolio behavior. Review of Financial Studies, 9(1):141-161.

Liu, J. (2007). Portfolio selection in stochastic environments. Review of Financial Studies, 20(1):1-39.

Merton, R. C. (1971). Optimum consumption and portfolio rules in a continuous time model. Journal of Economic Theory, 3:373-413. 


\section{Appendices}

\section{A.1 Discretization of continuous VAR model}

We aggregate the continuous VAR model over a span of time taking point observations at evenly spaced points $\left\{t_{0}, t_{1}, \ldots, t_{n}, t_{n+1}, \ldots\right\}$ with $\Delta t=t_{n}-t_{n-1}$. Thus, following Bergstrom (1984), the discrete version of our equation (8) becomes

$$
Y_{t_{n}+\Delta t}^{D}=\mathrm{e}^{(\Delta t \mathrm{G})} Y_{t_{n}}^{D}+U_{t_{n}+\Delta t}^{D}
$$

where

$$
U_{t_{n}+\Delta t}^{D}=\left[\begin{array}{c}
U_{t_{n}+\Delta t}^{p} \\
U_{t_{n}+\Delta t}^{x}
\end{array}\right]=\int_{\tau=0}^{\Delta t} \mathrm{e}^{(\Delta t-\tau) \mathrm{G}} \mathrm{H} \mathrm{d} Z_{t_{n}+\tau} .
$$

The matrices $\mathrm{G}$ and $\mathrm{H}$ are defined in (9) such that

$$
\mathrm{G}=\left[\begin{array}{cc}
0 & \sigma \\
0 & -\kappa
\end{array}\right] \text { and } \mathrm{H}=\left[\begin{array}{cc}
\sigma & 0 \\
\zeta \rho & \zeta \sqrt{1-\rho^{2}}
\end{array}\right]
$$

To derive analytical discrete version of (36), we need to evaluate two exponential forms, those involve square matrices. Starting by iterating forward integer powers of the matrix $\mathrm{G}$, we obtain

$$
\mathrm{G}^{n+1}=\left[\begin{array}{cc}
0 & (-\kappa)^{n} \sigma \\
0 & (-\kappa)^{n+1}
\end{array}\right]
$$

Since $\mathrm{G}$ is a square matrix, using Taylor series expansion of an exponential function, we can write:

$$
\mathrm{I}+\frac{\mathrm{G}^{1}}{1 !}+\frac{\mathrm{G}^{2}}{2 !}+\cdots+\frac{\mathrm{G}^{n+1}}{(n+1) !}+\cdots=\mathrm{e}^{\mathrm{G}} \Longleftrightarrow \sum_{m=0}^{+\infty} \frac{\mathrm{G}^{m}}{m !}=\mathrm{e}^{\mathrm{G}}
$$

More details about such transformation could be found in Gradshteyn and Ryzhik (2007, p. 1074). A direct application of this formula, given an arbitrary constant $v$, leads to

$$
\sum_{m=0}^{+\infty} \frac{(\mathrm{G} v)^{m}}{m !}=\mathrm{e}^{\mathrm{G} v}
$$

then

$$
\begin{gathered}
\mathrm{I}+\sum_{m=1}^{+\infty} \mathrm{G}^{m} \frac{v^{m}}{m !}=\left[\begin{array}{ll}
1 & 0 \\
0 & 1
\end{array}\right]+\sum_{m=1}^{+\infty}\left[\begin{array}{cc}
0 & \frac{(-\kappa)^{m-1} v^{m} \sigma}{m !} \\
0 & \frac{(-\kappa)^{m} v^{m}}{m !}
\end{array}\right]= \\
{\left[\begin{array}{cc}
1 & \sum_{m=1}^{+\infty} \frac{(-\kappa)^{m-1} v^{m} \sigma}{m !} \\
0 & 1+\sum_{m=1}^{+\infty} \frac{(-\kappa)^{m} v^{m}}{m !}
\end{array}\right]=\left[\begin{array}{cc}
1 & \frac{-\kappa}{-\kappa} \sum_{m=1}^{+\infty} \frac{(-\kappa)^{m-1} v^{m} \sigma}{m !} \\
0 & \sum_{m=0}^{+\infty} \frac{(-\kappa)^{m} v^{m}}{m !}
\end{array}\right] .}
\end{gathered}
$$

Thus

$$
\mathrm{e}^{\mathrm{G} v}=\left[\begin{array}{cc}
1 & -\frac{\sigma}{\kappa} \sum_{m=1}^{+\infty} \frac{(-\kappa v)^{m}}{m !} \\
0 & \sum_{m=0}^{+\infty} \frac{(-\kappa v)^{m}}{m !}
\end{array}\right] .
$$


It directly follows that

$$
\mathrm{e}^{\mathrm{G} v}=\left[\begin{array}{cc}
1 & \frac{\sigma}{\kappa}\left(1-\mathrm{e}^{-\kappa v}\right) \\
0 & \mathrm{e}^{-\kappa v}
\end{array}\right] .
$$

The last expression involving $\mathrm{e}^{\mathrm{G} v}$ holds for every constant $v$. Thus, substituting it into equation (36), equations (13) and (14) follow.

\section{A.2 Proof of continuous VAR recovering by discrete VAR}

The matrix (15) could be rewritten as

$$
\begin{aligned}
\Delta \ln P_{t+\Delta t}-r^{f} & =a_{r}+b_{r} z_{t}+\varepsilon_{t+\Delta t}^{r}, \\
z_{t+\Delta t} & =a_{z}+b_{z} z_{t}+\varepsilon_{t+\Delta t}^{z} .
\end{aligned}
$$

Equations (44) and (45) describe a bivariate econometric model in which $z$ denotes the log dividendprice ratio. The corresponding discretized version of the continuous-time model in matrix (13) could be rewritten as

$$
\begin{aligned}
\Delta \ln P_{t_{n}+\Delta t}-r \Delta t & =\left(\sigma \theta-\sigma^{2} / 2\right) \Delta t-\left(1-\mathrm{e}^{-\kappa \Delta t}\right) \frac{\sigma \theta}{\kappa}+\left(1-\mathrm{e}^{-\kappa \Delta t}\right) \frac{\sigma}{\kappa} X_{t_{n}}+U_{t_{n}+\Delta t}^{p}, \\
X_{t_{n}+\Delta t} & =\left(1-\mathrm{e}^{-\kappa \Delta t}\right) \theta+\mathrm{e}^{-\kappa \Delta t} X_{t_{n}}+U_{t_{n}+\Delta t}^{x} .
\end{aligned}
$$

Comparing the expectations of (44) and (46), we get

$$
z_{t}=-\frac{a_{r}}{b_{r}}+\frac{\sigma \theta-\sigma^{2} / 2}{b_{r}} \Delta t-\left(1-\mathrm{e}^{-\kappa \Delta t}\right) \frac{\sigma \theta}{b_{r} \kappa}+\left(1-\mathrm{e}^{-\kappa \Delta t}\right) \frac{\sigma}{b_{r} \kappa} X_{t_{n}} .
$$

Iterating forward (48) $\Delta t$ periods ahead and using (45), we obtain

$$
\begin{aligned}
& -\frac{a_{r}}{b_{r}}+\frac{\sigma \theta-\sigma^{2} / 2}{b_{r}} \Delta t-\left(1-\mathrm{e}^{-\kappa \Delta t}\right) \frac{\sigma \theta}{b_{r} \kappa}+\left(1-\mathrm{e}^{-\kappa \Delta t}\right) \frac{\sigma}{b_{r} \kappa} X_{t_{n}+\Delta t}= \\
& a_{z}+b_{z}\left(\frac{\sigma^{2} / 2-\sigma \theta}{b_{r}} \Delta t+\left(1-\mathrm{e}^{-\kappa \Delta t}\right) \frac{\sigma \theta}{b_{r} \kappa}-\left(1-\mathrm{e}^{-\kappa \Delta t}\right) \frac{\sigma}{b_{r} \kappa} X_{t_{n}}\right)+\varepsilon_{t+\Delta t}^{z}
\end{aligned}
$$

After some algebra, we find that

$$
X_{t_{n}+\Delta t}=-\left[\frac{a_{z} b_{r}}{\sigma}+\left(1-b_{z}\right)\left(\theta+\frac{a_{r}}{\sigma}-(\theta-\sigma / 2) \Delta t\right)\right] \frac{\kappa}{1-\mathrm{e}^{-\kappa \Delta t}}+b_{z} X_{t_{n}}-\frac{\kappa}{1-\mathrm{e}^{-\kappa \Delta t}} \frac{b_{r}}{\sigma} \varepsilon_{t+\Delta t}^{z} .
$$

Notice that $\lim _{\kappa \Delta t \rightarrow 0}\left(1-\mathrm{e}^{-\kappa \Delta t}\right)=\kappa \Delta t$. As a matter of fact, comparing equation (50) to (47), equations (16)-(18) directly follow. To compute the associated second moments, one can compute the variance of $U$ vector in (14).

$$
\mathrm{V}\left[\begin{array}{c}
U_{t_{n}+\Delta t}^{p} \\
U_{t_{n}+\Delta t}^{x}
\end{array}\right]=\int_{\tau=0}^{\Delta t} \mathrm{FF}^{\prime}\left[\begin{array}{l}
\mathrm{d} \tau \\
\mathrm{d} \tau
\end{array}\right]
$$

where

$$
\mathrm{F}=\left[\begin{array}{cc}
1 & \left(1-\mathrm{e}^{-\kappa \Delta t}\right) \frac{\sigma}{\kappa} \\
0 & \mathrm{e}^{-\kappa \Delta t}
\end{array}\right]\left[\begin{array}{cc}
\sigma & 0 \\
\zeta \rho & \zeta \sqrt{1-\rho^{2}}
\end{array}\right]
$$

Using a matching procedure involving equations (44)-(51), we could directly reset equations (19)-(21). Furthermore, when the hypothetical points $t_{n}=t$, the resulting conditional second moments for 
every $\Delta t$ become

$$
\begin{aligned}
\mathrm{V}\left(X_{t+\Delta t}\right)= & \frac{\zeta^{2}}{2 \kappa}\left(1-\mathrm{e}^{-2 \kappa \Delta t}\right), \\
\operatorname{Cov}\left(X_{t+\Delta t}, \Delta \ln P_{t+\Delta t}\right)= & \frac{\rho \sigma \zeta}{\kappa}\left(1-\mathrm{e}^{-\kappa \Delta t}\right)+\frac{\sigma \zeta^{2}}{\kappa^{2}}\left(1-\mathrm{e}^{-\kappa \Delta t}\right)-\frac{\sigma \zeta^{2}}{2 \kappa^{2}}\left(1-\mathrm{e}^{-2 \kappa \Delta t}\right), \\
\mathrm{V}\left(\Delta \ln P_{t+\Delta t}\right)= & \left(\sigma^{2}+2 \rho \frac{\zeta \sigma^{2}}{\kappa}+\frac{\zeta^{2} \sigma^{2}}{\kappa^{2}}\right) \Delta t-2 \rho \frac{\zeta \sigma^{2}}{\kappa^{2}}\left(1-\mathrm{e}^{-\kappa \Delta t}\right) \\
& -2 \frac{\zeta^{2} \sigma^{2}}{\kappa^{3}}\left(1-\mathrm{e}^{-\kappa \Delta t}\right)+\frac{\zeta^{2} \sigma^{2}}{2 \kappa^{3}}\left(1-\mathrm{e}^{-2 \kappa \Delta t}\right),
\end{aligned}
$$

where the instantaneous standard deviation of $X$ denoted $\zeta$ is given by equation (20). Again, notice that, for small $\kappa$, i.e. when $\kappa \Delta t \rightarrow 0$, the term $\left(1-\mathrm{e}^{-\kappa \Delta t}\right) \rightarrow \kappa \Delta t$. So when $\Delta t=1$, all conditional second moments could be approximated by their instantaneous counterparts. Otherwise, when $\Delta t \neq 1$, these kinds of approximations become no longer valid. Campbell et al. (2004, p. 2208) discuss about pitfalls resulting for this case. Taking this into account, for instance, one could compute the terminal conditional variances by just setting $\Delta t=T$ and $t=0$.

The unconditional moments of $X$ that have been used in this paper are derived from equation (48) when $\Delta t$ is normalized to one.

$$
\begin{aligned}
X_{t_{n}} & =\frac{\sigma}{2}+\frac{a_{r}+b_{r} z_{t}}{\sigma}, \\
\mathrm{E}\left(X_{t_{n}}\right) & =\frac{\sigma}{2}+\frac{a_{r}+b_{r} \mathrm{E}\left(z_{t}\right)}{\sigma}=\frac{\sigma}{2}+\frac{a_{r}+b_{r} a_{z} /\left(1-b_{z}\right)}{\sigma} .
\end{aligned}
$$

Hence, the unconditional mean of $X$ is

$$
\theta=\frac{a_{z} b_{r}}{\sigma_{r}\left(1-b_{z}\right)}+\frac{a_{r}+\sigma_{r}^{2} / 2}{\sigma_{r}} .
$$

In fact, we have used the result $\sigma=\sigma_{r}$ in equation (19) and $z_{t}$ follows an AR(1) process (Brandt et al. (2005) followed by Garlappi and Skoulakis (2009) among others). Thus, the unconditional moments of $z_{t}$ are known, $\mathrm{E}\left(z_{t}\right)=a_{z} /\left(1-b_{z}\right)$ and $\mathrm{V}\left(z_{t}\right)=\sigma_{z}^{2} /\left(1-b_{z}^{2}\right)$ (Hamilton $(1994, \mathrm{p} .53)$ ). So, under equation (48), one can match all unconditional percentiles $z_{(p)}$ with their unconditional counterparts $X_{(p)}$ (where $p$ denotes the $p$-th percentile), and get optimal policies for those values. We directly draw $X_{(p)}$ from the unconditional distribution of the point observations $X_{t}$ of our continuous-time state variable $X$. Thus, $X_{(50)}=\theta$, and the following unconditional distributions hold:

$$
z \sim \mathrm{N}\left(\frac{a_{z}}{1-b_{z}}, \frac{\sigma_{z}^{2}}{1-b_{z}^{2}}\right) \Longrightarrow X \sim \mathrm{N}\left(\theta, \frac{b_{r}^{2}}{1-b_{z}^{2}} \frac{\sigma_{z}^{2}}{\sigma_{r}^{2}}\right)
$$

\section{A.3 Derivation of parameters $C_{1}$ and $C_{2}$}

Regarding (33), the solution for $C_{2}$ could be derived from the equation

$$
\frac{\mathrm{d} C_{2}}{\mathrm{~d} t}+a C_{2}^{2}+b C_{2}+c=0 .
$$

This equation could straightforward be rewritten as

$$
\int_{t}^{T} \frac{1}{a C_{2}^{2}+b C_{2}+c} \mathrm{~d} C_{2}=-(T-t) .
$$


Since parameters $a, b$ and $c$ are constant over time, given $C_{2}(T)=0$, integral table provides the solution for $C_{2}$ as in (34). Substitute this into the following equation

$$
\frac{\mathrm{d} C_{1}}{\mathrm{~d} t}+\kappa \theta C_{2}+\left(\frac{b}{2}+a C_{2}\right) C_{1}=0
$$

that we derived from equation (33). Again using the terminal condition $C_{1}(T)=0$ and the constant variation method, we get the solution for $C_{1}$ as reported in (35). 